\title{
COUMARINS FROM THE ROOTS OF Prangos
}

quasiperforata

L. I. Shagova, M. G. Pimenov, and G. A. Kuznetsova

UDC 547.814

We have studied the coumarin composition of the roots of Prangos quasiperforata collected in Kirghizia (R. Alabuga, Koshtobe). An ethanolic extract was chromatographed on a column of neutral alumina (Brockman activity grade III) and was eluted with petroleum ether, mixtures of petroleum ether and chloroform (3:1 and $1: 1)$, chloroform, and ethanol. The following coumarins were isolated and were identified by their melting points, mixed melting points with authentic samples, and IR spectra: osthole, isoimperatorin, oxypeucedanin, oxypeucedanin nitrate, and for the first time, (-)-prangenin hydrate, $\mathrm{mp} 127^{\circ} \mathrm{C},[\alpha]_{\mathrm{D}}^{21}-9^{\circ}$ (c 1.3 ; ethanol).

Several other coumarin derivatives were detected by paper chromatography and gas-liquid chromatography (GLC). Paper chromatography showed the presence of deltoin, pranchimgin, imperatorin, bergapten, prangenin, psoralen, marmesin, and two unidentified compounds: (I) with $\mathrm{R}_{f} 0.05$ (blue) and (II) with $\mathrm{R}_{f} 0.20$ (yellow) [system (I): mobile phase petroleum ether, stationary phase ethylene glycol]. GLC was performed on a Pye-Unicam 104 instrument using a column $(l 1.5 \mathrm{~m})$ containing the stationary phase OV-17 $(3 \%)$ on Chromosorb W (50-60 mesh). The temperature was programmed at $2^{\circ} \mathrm{C} / \mathrm{min}, 180-256^{\circ} \mathrm{C}$. The rate of flow of the carrier gas, argon, was $30 \mathrm{ml} / \mathrm{min}$. The following were identified: psoralen (Fig. 1, peak 2), bergapten (4), osthole (5), marmesin (6), deltoin (9), and pranchimgin (11), and also for the first time in plants of the genus Prangos we detected three substances with RRTs (relative retention times calculated with respect to osthole) of 0.4 (1), $0.6(3)$, and $4.44(10)$. Under these conditions of GLC, the prangenin and oxypeucedanin and their hydrates (7, 8 ) are not separated.

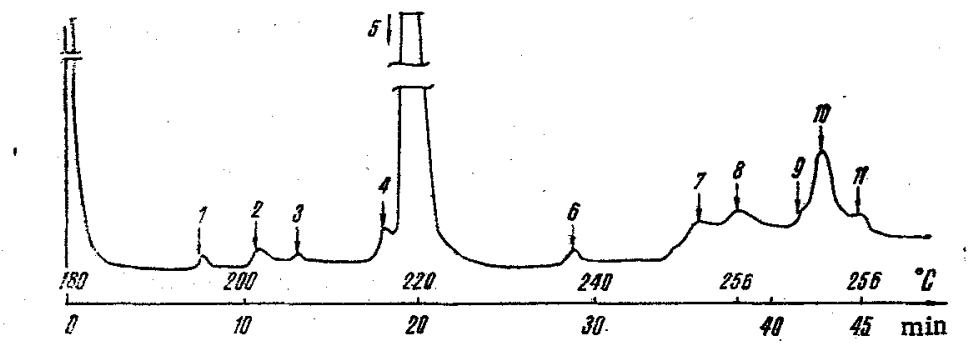

Fig. 1. Chromatogram of an extract of the roots of Prangos quasiperforata Kuzm: 1) unidentified compound; 2) psoralen; 3) unidentified compound; 4) bergapten; 5) osthole; 6) marmesin; 7, 8) prangenin + oxypeucedanin + prangenin hydrate + oxypeucedanin hydrate; 9) deltoin; 10) unidentified compound; 11) pranchimgin.

M. V. Lomonosov Moscow State University. V. L. Komarov Botanical Institute, Academy of Sciences of the USSR, Leningrad. Translated from Khimiya Prirodnykh Soedinenii, No. 3, pp. 386-387, May-June, 1976. Original article submitted December 10, 1975.

This material is protected by' copyright registered in the name of Plenum Publishing Corporation, 227 West 17th Street, New York, N.Y. 10011. No part of this publication may be reproduced, stored in a retrieval system, or transmitted, in any form or by any means, electronic, mechanical, photocopying, microfilming, recording or otherwise, without written permission of the publisher. A copy of this article is available from the publisher for $\$ 7.50$. 